\title{
Effect of Acetazolamide on Chemosensitivity, Cheyne-Stokes Respiration, and Response to Effort in Patients With Heart Failure
}

\author{
Marianna Fontana, $\mathrm{MD}^{\mathrm{a}}$, Michele Emdin, $\mathrm{MD}, \mathrm{PhD}^{\mathrm{a}}$, Alberto Giannoni, $\mathrm{MD}^{\mathrm{a}, \mathrm{b}}$, \\ Giovanni Iudice, $\mathrm{BSc}^{\mathrm{a}}$, Resham Baruah, $\mathrm{MD}^{\mathrm{c}}$, and Claudio Passino, $\mathrm{MD}^{\mathrm{a}, \mathrm{b}, *}$
}

\begin{abstract}
Increased chemosensitivity to hypoxia and hypercapnia, together with a prolonged circulatory time, are the main determinants of Cheyne-Stokes (C-S) respiration in heart failure. To evaluate the effect of acetazolamide, a carbonic anhydrase inhibitor, on chemosensitivity and respiratory dynamics in patients with heart failure with $\mathrm{C}-\mathrm{S}$ respiration, 12 patients (mean age $62 \pm 9$ years, mean left ventricular ejection fraction $24 \pm 9 \%$ ) and C-S respiration (mean apnea-hypopnea index $23 \pm 13$ ) who underwent 4 consecutive days of oral acetazolamide treatment ( $250 \mathrm{mg}$ twice daily) were enrolled in this study. Assessment of chemosensitivity to hypoxia and hypercapnia, cardiopulmonary stress testing, 24-hour cardiorespiratory polygraphy, and neurohormonal characterization were performed at baseline and at the end of treatment. Acetazolamide improved central apneas (apneahypopnea index $23 \pm 13$ to $15 \pm 9, p=0.012$ ) and the percentage of time spent below an arterial oxyhemoglobin saturation of $90 \%(16 \pm 23 \%$ to $10 \pm 18 \%, p=0.005)$. Chemosensitivity to hypoxia was blunted $(1.03 \pm 0.69$ to $0.78 \pm 0.55 \mathrm{~L} / \mathrm{min} / \mathrm{mm} \mathrm{Hg}, \mathrm{p}=0.032)$, while chemosensitivity to hypercapnia increased after acetazolamide $(1.27 \pm 0.71$ to $1.54 \pm 0.78 \mathrm{~L} / \mathrm{min} / \%$ arterial oxygen saturation, $p=0.023$ ); patients achieved a lower workload $(90 \pm 30$ to $81 \pm 30 \mathrm{~W}, \mathrm{p}<0.001)$, with no differences in peak oxygen consumption, while there was an increment in the regression slope relating minute ventilation to carbon dioxide output ( $39 \pm 10$ to $43 \pm 9, p=0.010)$. In conclusion, in patients with heart failure, acetazolamide diminishes $\mathrm{C}-\mathrm{S}$ respiration and improves oxyhemoglobin saturation, likely by decreasing chemosensitivity to hypoxia. However, it is associated with reduced maximal workload achieved during effort and increased chemosensitivity to hypercapnia, inducing a reduction in the ventilatory efficiency. (c) 2011 Elsevier Inc. All rights reserved. (Am J Cardiol 2011;107:1675-1680)
\end{abstract}

Some studies have reported a beneficial effect of acetazolamide, a carbonic anhydrase inhibitor, in the treatment of central sleep apnea at high altitude ${ }^{1,2}$ and in idiopathic central sleep apnea. ${ }^{3,4}$ However, only 1 study has demonstrated acetazolamide to be effective also in patients with heart failure (HF) with Cheyne-Stokes (C-S) respiration, ${ }^{5}$ although the exact mechanisms through which this occurred remain unclear. Increased chemosensitivity to hypercapnia ${ }^{6}$ and hypoxia ${ }^{7}$ contributes to trigger the onset and perpetuate $\mathrm{C}-\mathrm{S}$ respiration in patients with chronic HF. ${ }^{8,9}$ Furthermore, chemosensitivity is thought to exert a direct and independent action on the adrenergic drive, ${ }^{10,11}$ leading to ventricular arrhythmias, ${ }^{6,8,11,12}$ ventilatory inefficiency during exercise, ${ }^{11,13}$ and, most important, overall worse prognosis. ${ }^{14}$ Studies in healthy subjects suggest that acetazolamide may directly affect chemosensitivity, increasing sensitivity to hypercapnia and decreasing sensitivity to hypoxia. ${ }^{15-18} \mathrm{We}$ hypothesized that acetazolamide may affect chemosensitivity to hypoxia and hypercapnia in patients with HF, a hy-

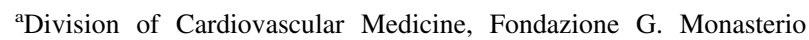
CNR-Regione Toscana; 'Scuola Superiore Sant'Anna, Pisa, Italy; and 'International Centre for Circulatory Health, St. Mary's Hospital and Imperial College, London, United Kingdom. Manuscript received December 9, 2010; revised manuscript received and accepted January 22, 2011.

*Corresponding author: Tel: 39-050-3152191; fax: 39-050-3152109.

E-mail address: passino@ftgm.it (C. Passino). pothesis that has never been investigated in this setting, this being 1 possible mechanism for its efficacy in reducing the incidence of C-S respiration. We therefore evaluated the effect of acetazolamide on chemosensitivity to hypoxia and hypercapnia, as well as its effect on respiratory pattern at rest and during exercise.

\section{Methods}

From September 2008 to February 2010, we screened 48 ambulatory patients with HF from our clinic with left ventricular systolic dysfunction (left ventricular ejection fraction $<50 \%$ ) and $\mathrm{C}-\mathrm{S}$ respiration on ambulatory cardiorespiratory polygraphy (apnea-hypopnea index, defined as the number of apnea or hypopnea episodes per hour of recording, $>15$ per hour). Exclusion criteria were New York Heart Association class IV, acute coronary syndromes within the previous 6 months, glomerular filtration rate $<30 \mathrm{ml} / \mathrm{min}$, pulmonary disease defined as vital capacity and total lung capacity $<50 \%$ of predicted value, forced expiratory volume in 1 second $<50 \%$ of predicted value, and forced vital capacity $<70 \%$. Patients with obstructive sleep apnea syndrome, defined as a number of obstructive events $>50 \%$ of total hypopnea episodes, and current treatment with morphine or derivatives, theophylline, oxygen, benzodiazepines, or acetazolamide were also excluded. Twelve patients matched the entry criteria and were enrolled in the 


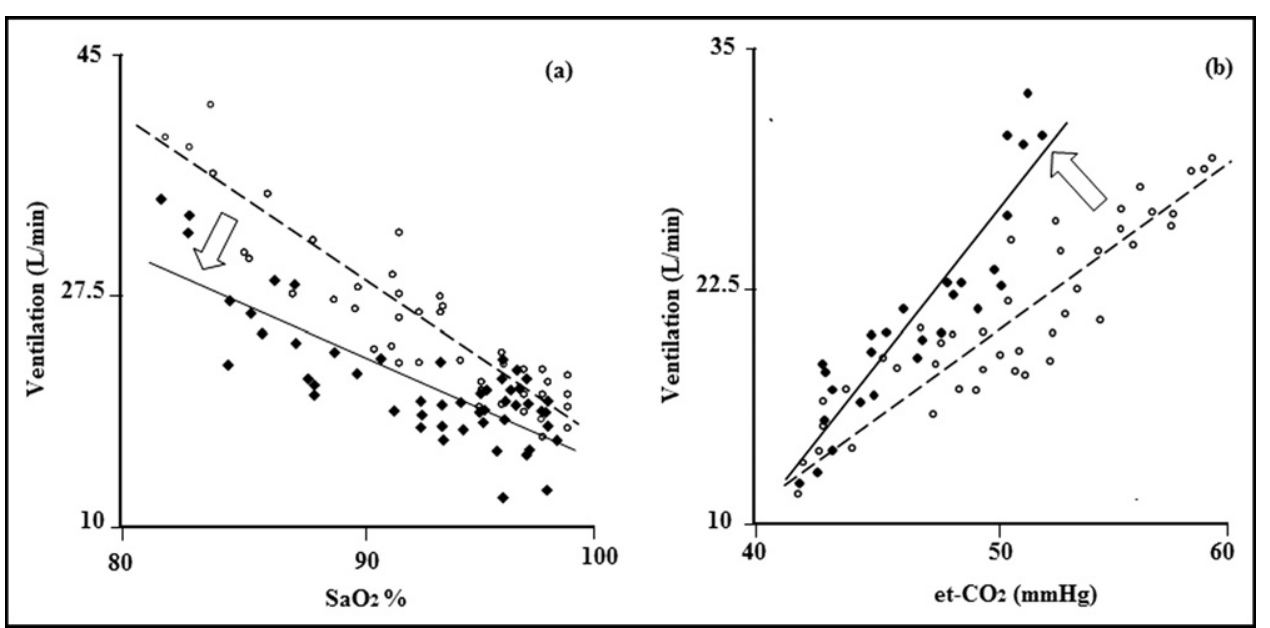

Figure 1. Results of rebreathing tests during hypoxic normocapnic trial $(a)$ and hypercapnic-normoxic trial $(b)$ in a representative subject. Changes in breath-by-breath ventilation are shown before (open circles) and after (solid circles) acetazolamide administration.

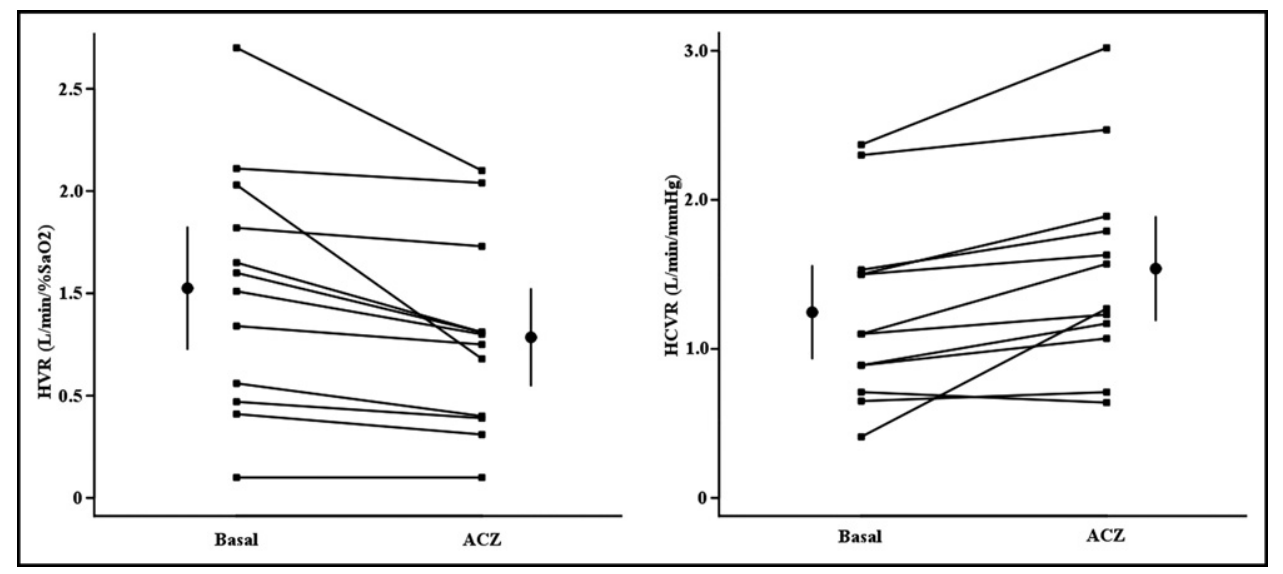

Figure 2. Individual changes in chemosensitivity during hypoxic normocapnic trial (left) and hypercapnic-normoxic trial (right) before and after acetazolamide administration. Each subject is represented by a black square. Solid black circles represent the average values before and after treatment.

study. In addition to the standard therapeutic regimen, patients were treated with oral acetazolamide $250 \mathrm{mg}$ twice daily for 4 days. A 4-day treatment regimen was chosen to assess the "long-term" effect of acetazolamide administration, while the double administration was chosen to study the effect of the drug on both nighttime and daytime C-S respiration.

At baseline and at the end of treatment, all patients underwent (1) evaluation of chemosensitivity; (2) blood sampling for N-terminal-pro-brain natriuretic peptide, epinephrine, norepinephrine, plasma renin activity, and aldosterone; (3) arterial blood gas analysis; (4) cardiopulmonary exercise stress testing; and (5) 24-hour ambulatory cardiorespiratory polygraphy.

The protocol was approved by the local ethics committee. Informed consent was obtained from all subjects.

Chemosensitivity was assessed using the rebreathing technique, as previously described. ${ }^{11,13}$ Chemosensitivity to hypoxia, or hypoxic ventilatory response (HVR), was calculated as the linear regression slope between minute ventilation and arterial oxygen saturation during the hypoxicnormocapnic trial. Chemosensitivity to hypercapnia, or hypercapnic ventilatory response (HCVR), was calculated as the linear regression slope between minute ventilation and end-tidal carbon dioxide during the hypercapnicnormoxic trial. As cut-off values for defining increased chemosensitivity, we considered $0.77 \mathrm{~L} / \mathrm{min} / \mathrm{mm} \mathrm{Hg}$ for HVR and $0.79 \mathrm{~L} / \mathrm{min} / \%$ arterial oxygen saturation for HCVR (i.e., 2 times the standard deviation over the mean value of a control group of healthy subjects). ${ }^{11}$ All patients were familiar with chemosensitivity testing.

Neurohormonal assays were performed as described elsewhere. ${ }^{19}$ Symptom-limited cardiopulmonary exercise tests were conducted on a bicycle ergometer according to a ramp protocol $\left(\mathrm{V}_{\max }\right.$; Sensormedics, Milan, Italy). Circulatory power was calculated as peak oxygen uptake $\left(\mathrm{VO}_{2}\right)$ multiplied by peak systolic blood pressure, ${ }^{20}$ and peak oxygen pulse was calculated as peak $\mathrm{VO}_{2}$ divided by heart rate. All cardiopulmonary exercise tests were performed by the same physician, blinded to the results of the chemosensitivity tests. All patients were familiar with cardiopulmonary exercise testing. All subjects underwent a 24-hour continuous ambulatory polygraphic recording that included thoracic and abdominal respiratory inductance plethysmography, airflow (using a nasal-oral thermocouple and nasal pressure recording), finger pulse oximetry, and electrocar- 


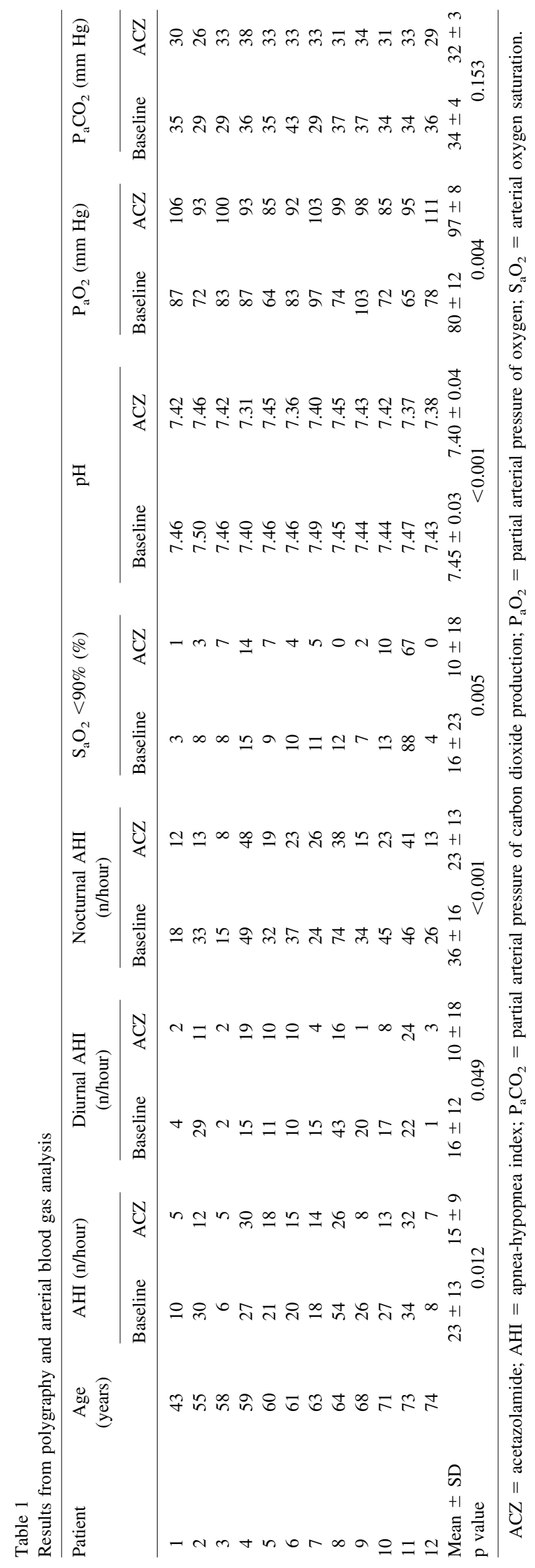

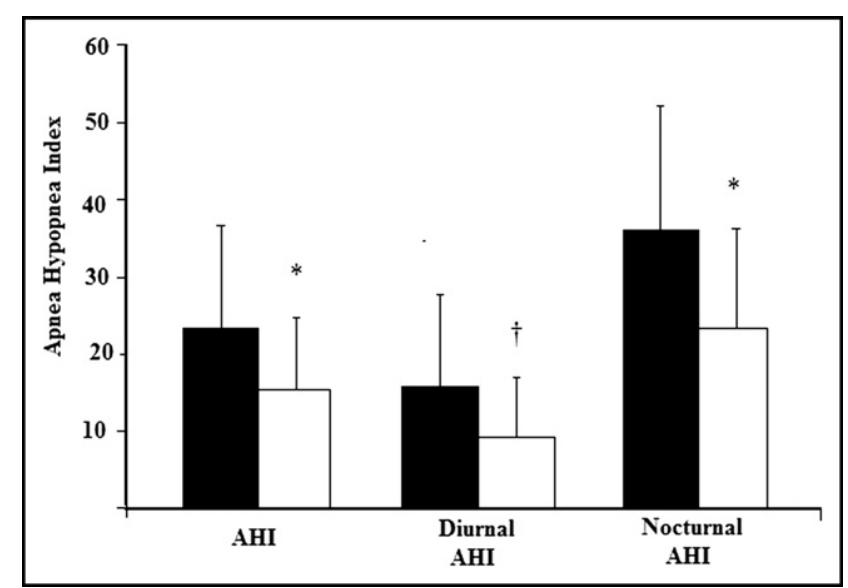

Figure 3. Changes in the 24-hour, diurnal, and nocturnal apnea-hypopnea index (AHI) are shown before (black) and after (white) acetazolamide administration. " $\mathrm{p}<0.001$ and ${ }^{\dagger} \mathrm{p}<0.05$, acetazolamide compared to baseline.

diography (Somte; Compumedics, Abbotsford, Australia). An episode of apnea was defined as the cessation of respiratory flow for $\geq 10$ seconds, whereas hypopnea was defined as a reduction in respiratory flow lasting $\geq 10$ seconds and associated with $\mathrm{a} \geq 4 \%$ decrease in arterial oxyhemoglobin saturation. ${ }^{21}$ Apnea and hypopnea were considered as central or obstructive according to the combined analysis of ribcage and abdominal excursions and airflow signal.

Statistical analysis was performed using SPSS version 13.0 (SPSS, Inc., Chicago, Illinois). Data are expressed as mean \pm SD if normally distributed or as median (interquartile range) otherwise. Neurohormones were logarithmically transformed to normalize a skewed distribution when needed. Paired Student's $t$ tests or McNemar's tests were performed to compare the effect of acetazolamide with baseline status within subjects. The sample size was determined for the principal question, namely, changes in chemoreflex sensitivity after acetazolamide administration. In a pilot study with 3 patients, we found that 12 patients would be sufficient to reach $80 \%$ power to detect changes in HCVR and HVR. A p value $<0.05$ was considered statistically significant.

\section{Results}

The study population consisted of 12 patients with HF (11 men) receiving optimal medical therapy. The mean age was $62 \pm 9$ years, the mean body mass index $29 \pm 4 \mathrm{~kg} / \mathrm{m}^{2}$, and the mean left ventricular ejection fraction $24 \pm 9 \%$. On the whole, patients showed at baseline a moderate degree of neurohormonal activation: median $\mathrm{N}$-terminal-pro-brain natriuretic peptide was $1,216 \mathrm{ng} / \mathrm{L}$ (interquartile range 863 to 1,844 ), median norepinephrine $509 \mathrm{ng} / \mathrm{L}$ (interquartile range 461 to 736), median plasma renin activity $2.49 \mathrm{ng} / \mathrm{L} /$ hour (interquartile range 1.71 to 4.03 ), and median aldosterone $264 \mathrm{ng} / \mathrm{L}$ (interquartile range 122 to 279 ).

At baseline, 8 patients demonstrated elevated chemosensitivity with increases in HVR and HCVR, 1 patient demonstrated an increase solely in HVR, and 3 patients had normal HVR and HCVR. 


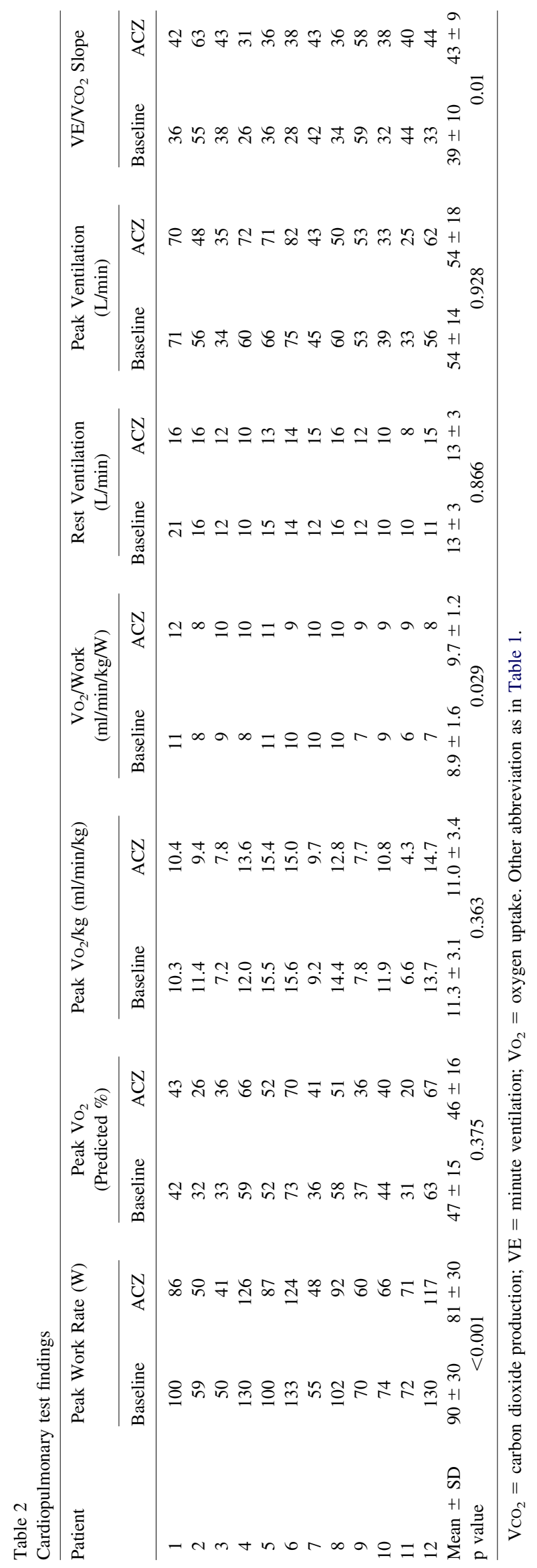

Administration of acetazolamide had different effects on chemosensitivity to hypoxia or hypercapnia (Figures 1 and 2). Indeed, while chemosensitivity to hypoxia was blunted by acetazolamide by $24 \%$ (HVR $1.03 \pm 0.69$ to $0.78 \pm 0.55$ $\mathrm{L} / \mathrm{min} / \mathrm{mm} \mathrm{Hg}, \mathrm{p}=0.03$; Figure 2), chemosensitivity to hypercapnia increased by $21 \%$ (HCVR $1.27 \pm 0.71$ to 1.54 $\pm 0.78 \mathrm{~L} / \mathrm{min} / \%$ arterial oxygen saturation, $\mathrm{p}=0.02$; Figure 2). With respect to arterial blood gas analysis, acetazolamide resulted in a mild metabolic acidosis, with a nonsignificant trend toward a reduction of the partial arterial pressure of carbon dioxide and a significant improvement in the partial arterial pressure of oxygen and oxyhemoglobin saturation (Table 1).

All patients had central sleep apnea, but 3 patients also had from obstructive events (relative percentage of obstructive events $43 \%, 22 \%$, and $2 \%$ ). Acetazolamide $250 \mathrm{mg}$ twice daily significantly reduced central sleep apnea (Table 1). This stabilization of ventilation with acetazolamide was not confined solely to central sleep apnea at night; there was also a significant reduction in daytime $\mathrm{C}-\mathrm{S}$ respiration, with a $41 \%$ reduction of diurnal apnea-hypopnea index (Figure 3 ). The observed improvement in apnea-hypopnea index was due to a reduction in central events, because there was no decrease in the number of obstructive events in the 3 patients with episodes of obstructive sleep apnea. The reduction in central events was associated with a significant improvement in arterial oxyhemoglobin saturation, as reflected by a $36 \%$ decrease of the total time spent with a saturation $<90 \%$ (Table 1) and by an increase in the minimal oxygen saturation achieved during apneas $(82 \pm 5$ to $86 \pm 3, \mathrm{p}=0.001)$.

At baseline, the main limitation to exercise was dyspnea, as reported by 9 patients, whereas after acetazolamide administration, leg fatigue became the predominant limiting symptom, which was reported by 9 patients. Exertional oscillatory ventilation was demonstrated in 6 of 12 patients; its prevalence was unchanged by 4 days of treatment with acetazolamide. Exercise capacity after acetazolamide administration was reduced by $10 \%$, with no change in peak $\mathrm{VO}_{2}$ reached. Acetazolamide was associated with a $13 \%$ increase in the regression slope relating ventilation to carbon dioxide production (minute ventilation/carbon dioxide production slope), despite no difference in peak ventilation (Table 2). Maximal heart rate and blood pressure were lower at peak exercise on acetazolamide, with a corresponding significant reduction in the rate-pressure product (all $\mathrm{p}$ values $<0.05$ ), while there was no difference in the circulatory power and in the peak oxygen pulse (data not shown).

Despite an increase in hypercapnic chemosensitivity after acetazolamide, there was no clear change in sympathetic activation, heart rate at rest, systolic and diastolic blood pressure, and plasma norepinephrine, N-terminal-pro-brain natriuretic peptide, plasma renin activity, and aldosterone concentration (data not shown).

\section{Discussion}

This study demonstrates that in patients with HF and C-S respiration, acetazolamide blunts chemosensitivity to hypoxia while increasing the chemosensitivity to hypercapnia. 
This action on the chemoreflex is associated with (1) significant decreases in nighttime and daytime $\mathrm{C}-\mathrm{S}$ respiration and (2) improvement in oxygen saturation, with no adverse impact on sympathetic activation. However, acetazolamide was associated with a negative effect on maximal workload achieved during exercise and on ventilatory efficiency, with no change in peak $\mathrm{VO}_{2}$.

The effectiveness of acetazolamide on central sleep apnea in patients with HF has previously been demonstrated ${ }^{5}$; however, the present study extends this finding to daytime periodic breathing and proposes a mechanism of action. The half-life of acetazolamide means that, when administered twice a day, its effects can be demonstrated over a full 24-hour period, unlike other treatment strategies, such as noninvasive mechanical ventilation or supplemental overnight oxygen therapy.

Experimental evidence on $\operatorname{dog} \mathrm{s}^{22}$ has suggested that acetazolamide might exert its positive action on C-S respiration by increasing the gap between the apneic threshold and the prevailing carbon dioxide. However, a direct effect of acetazolamide on chemoreflex sensitivity, which plays a critical role in the onset of C-S respiration in patients with $\mathrm{HF},{ }^{6-8}$ may also contribute the beneficial actions of acetazolamide. The blunting effect on chemosensitivity to hypoxia and the stimulation of chemosensitivity to hypercapnia elicited by acetazolamide, demonstrated for the first time in patients with HF in our study, is consistent with previous reports in healthy subjects. ${ }^{15-18}$ Central chemosensitivity is believed to be responsible for ventilation and carbon dioxide set-point determination, while peripheral chemosensitivity to oxygen and carbon dioxide favors the fluctuation of ventilation and respiratory gases around their mean set points. ${ }^{8}$ Indeed, the blunting effects of acetazolamide on the hypoxic ventilatory response could be responsible for the improvement of periodic breathing observed after drug administration. Moreover, the improvement in oxyhemoglobin saturation (after the reduction in diurnal and nocturnal central apnea) could further limit the hypoxic stimulus for hyperventilation and positively modulate the carbon dioxide response of the peripheral chemoreceptors, ${ }^{23}$ favoring the development of a virtuous cycle that stabilizes periodic breathing.

There is growing evidence that the enhancement of chemosensitivity to hypercapnia, a powerful and independent predictor of mortality in patients with HF, may play an unfavorable role in HF progression through an increase in sympathetic drive, ${ }^{10}$ eliciting autonomic imbalance, neurohormonal activation, abnormal ventilatory responses, and ventricular arrhythmias. ${ }^{11,14}$ The observation of increased HCVR and of a neutral effect on neurohormonal activation after treatment with acetazolamide raises some concerns regarding potential negative effects of long-term acetazolamide administration.

We found a $10 \%$ reduction in workload achieved at maximal exercise level, with leg fatigue becoming the predominant limiting symptom. This is in accordance with previous reports in healthy subjects ${ }^{18}$ and may be due to acetazolamide-induced mild acidosis that may decrease endurance time, through the inhibition of glycolytic enzymes, especially phosphofructokinase. ${ }^{24}$ Interestingly, whatever the mechanism of the reduction in individual workload, no difference in peak $\mathrm{VO}_{2}$ achieved at maximal exercise was seen. Moreover, the $\mathrm{VO}_{2}$ /work slope was higher after acetazolamide treatment, counterbalancing the effect of the reduced workload achieved on peak $\mathrm{VO}_{2}$. This effect could be due to the improvement in oxyhemoglobin saturation related to the disappearance or attenuation of C-S respiration and to a plausible right shift in the oxyhemoglobin dissociation curve due to acidosis (Bohr effect).

As far as respiratory dynamics is concerned, ventilation at rest was unchanged, while peak exercise ventilation increased on acetazolamide compared to baseline. The similar ventilation at rest could be related to the balance between an inhibitory effect of acetazolamide on the peripheral chemoreceptor and the stimulant effect on the central chemoreceptor. In contrast, the observed increased ventilation during exercise, associated with an increase in the minute ventilation/carbon dioxide production slope, may be related to the stimulating action of acetazolamide on the chemosensitivity to hypercapnia.

Finally, the net balance of acetazolamide (decreased chemosensitivity to hypoxia/increased sensitivity to hypercapnia) on sympathetic drive appears to be neutral, as indicated by the absence of significant changes in rest norepinephrine concentration before versus after treatment.

A major limitation of this study was the lack of a control group. However, all patients were familiar with cardiopulmonary exercise testing and the chemosensitivity evaluation, limiting intrapatient variability, and the individual changes observed in chemoreflex sensitivity after treatment were consistent in all patients, as shown in Figure 2, and in accordance with previous studies conducted in healthy subjects. Second, this was a short-term study, and studies specifically focused on long-term response to chronic acetazolamide administration are needed to highlight the long-term effects of acetazolamide on acid-base balance, sympathetic activation, exercise capacity and chemosensitivity. Third, plasma norepinephrine assay is only an approximate marker of sympathetic drive, but plasma norepinephrine level can be used for a rough estimation of the degree of sympathetic activation and has been proved to change in response to stimuli and drugs known to act on the sympathetic drive.

1. Sutton JR, Houston CS, Mansell AL, McFadden MD, Hackett PM, Rigg JR, Powles AC. Effect of acetazolamide on hypoxemia during sleep at high altitude. N Engl J Med 1979;301:1329-1331.

2. Hackett PH, Roach RC, Harrison GL, Schoene RB, Mills WJ Jr. Respiratory stimulants and sleep periodic breathing a high altitude: almitrine versus acetazolamide. Am Rev Respir Dis 1987;135:896-898.

3. White DP, Zwillich CS, Pickett CK, Douglas NJ, Findley LJ, Weil JV. Central sleep apnea improvement with acetazolamide therapy. Arch Intern Med 1982;182:1816-1819.

4. DeBacker WA, Verbraecken J, Willemen M, Wittesaele W, DeCock W, Van deHeynin P. Central apnea index decreases after prolonged treatment with acetazolamide. Am J Respir Crit Care Med 1995;151:87-91.

5. Javaheri S. Acetazolamide improves central sleep apnea in heart failure: a double-blind, prospective study. Am J Respir Crit Care Med 2006;173:234-237

6. Javaheri S. A mechanism of central sleep apnea in patients with heart failure. N Engl J Med 1999;341:949-954.

7. Ponikowski P, Anker SD, Chua TP, Francis D, Banasiak W, PooleWilson PA, Coats AJ, Piepoli M. Oscillatory breathing patterns during wakefulness in patients with chronic heart failure: clinical implications and role of augmented peripheral chemosensitivity. Circulation 1999; 100:2418-2424. 
8. Solin P, Roebuck T, Johns DP, Walters EH, Naughton MT. Peripheral and central ventilatory responses in central sleep apnea with and without congestive heart failure. Am J Respir Crit Care Med 2000; 162:2194-2200

9. Francis DP, Willson K, Davies LC, Coats AJ, Piepoli M. Quantitative general theory for periodic breathing in chronic heart failure and its clinical implications. Circulation 2000;102:2214-2221.

10. Narkiewicz K, Pesek CA, van de Borne PJ, Kato M, Somers VK. Enhanced sympathetic and ventilatory responses to central chemoreflex activation in heart failure. Circulation 1999;100:262-267.

11. Giannoni A, Emdin M, Poletti R, Bramanti F, Prontera C, Piepoli M, Passino C. Clinical significance of chemosensitivity in chronic heart failure: influence on neurohormonal derangement, Cheyne-Stokes respiration and arrhythmias. Clin Sci 2008;114:489-497.

12. Chua TP, Ponikowski P, Webb-Peploe K, Harrington D, Anker SD, Piepoli M, Coats AJ. Clinical characteristics of chronic heart failure patients with an augmented peripheral chemoreflex. Eur Heart J 1997; 18:480-486.

13. Chua TP, Clark AL, Amadi AA, Coats AJ. The relationship between chemosensitivity and the ventilatory response to exercise in chronic heart failure. J Am Coll Cardiol 1996;27:650-657.

14. Giannoni A, Emdin M, Bramanti F, Iudice G, Francis DP, Barsotti A, Piepoli M, Passino C. Combined increased chemosensitivity to hypoxia and hypercapnia as a prognosticator in heart failure. $J$ Am Coll Cardiol 2009;53:1975-1980.

15. Swenson ER \&amp; Hughes JMB. Effects of acute and chronic acetazolamide on resting ventilation responses in men. J Appl Physiol 1993;74:230-237.
16. Tojima H, Kunitomo F, Okita S, Yuguchi Y, Tatsumi K, Kimura H, Kuriyama T, Watanabe S, Honda Y. Difference in the effects of acetazolamide and ammonium chloride on ventilatory responses to $\mathrm{CO}_{2}$ and hypoxia in humans. Jpn J Physiol 1986;36:511-521.

17. Bashir Y, Kann M, Stradling JR. The effect of acetazolamide in hypercapnic and eucapnic-poikilocapnic hypoxic ventilatory responses in normal subjects. Pulm Pharmacol Ther 1990;3:151-154.

18. Garske LA, Brown MG, Morrison SC. Acetazolamide reduces exercise capacity and increases leg fatigue under hypoxic conditions. J Appl Physiol 2003;94:991-996.

19. Emdin M, Passino C, Prontera C, Iervasi A, Ripoli A, Masini S, Zucchelli GC, Clerico A. Cardiac natriuretic hormones, neurohormones, thyroid hormones and cytokines in normal subjects and patients with heart failure. Clin Chem Lab Med 2004;42:627-636.

20. Cohen-Solal A, Tabet JY, Logeart D, Bourgoin P, Tokmakova M, Dahan M. A non-invasively determined surrogate of cardiac power ("circulatory power") at peak exercise is a powerful prognostic factor in chronic heart failure. Eur Heart J 2002;23:806-814.

21. Yamashiro Y, Kryger MH. Sleep in heart failure. Sleep 1993;16:513523.

22. Nakayama H, Smith CA, Rodman JR, Skatrud JB, Dempsey JA. Effect of ventilatory drive on carbon dioxide sensitivity below eupnea during sleep. Am J Respir Crit Care Med 2002;165:1251-1259.

23. Duffin J. Role of acid-base balance in the chemoreflex control of breathing. J Appl Physiol 2005;99:2255-2265.

24. Jones NL, Sutton JR, Taylor R, Toews CJ. Effect of pH on cardiorespiratory and metabolic responses to exercise. J Appl Physiol 1977; 43:959-964. 